OPEN ACCESS

Edited by: Bas van Heur,

Vrije University Brussel, Belgium

Reviewed by:

Clancy Wilmott,

University of California, Berkeley,

United States

Alex Pazaitis,

Tallinn University of

Technology, Estonia

*Correspondence:

Martijn De Waal

b.g.m.de.waal@hva.nl

Specialty section

This article was submitted to Governance and Cities,

a section of the journal

Frontiers in Sustainable Cities

Received: 14 February 2020

Accepted: 05 October 2020

Published: 14 December 2020

Citation:

Gloerich I, De Waal M, Ferri G, Cila N and Karpinski $T$ (2020) The City as a License. Implications of Blockchain and Distributed Ledgers for Urban

Governance.

Front. Sustain. Cities 2:534942. doi: 10.3389/frsc.2020.534942

\section{The City as a License. Implications of Blockchain and Distributed Ledgers for Urban Governance}

\author{
Inte Gloerich ${ }^{1}$, Martijn De Waal ${ }^{1 *}$, Gabriele Ferri ${ }^{1}$, Nazli Cila ${ }^{2}$ and Tara Karpinski ${ }^{3}$ \\ ${ }^{1}$ Faculty of Digital Media and Creative Industries, Amsterdam University of Applied Sciences, Amsterdam, Netherlands, \\ ${ }^{2}$ Department of Human-Centered Design, Delft University of Technology, Delft, Netherlands, ${ }^{3}$ Avans University of Applied \\ Sciences, Centre of Expertise for Art, Design and Technology, Breda, Netherlands
}

Distributed ledger technologies (DLTs) such as blockchain have in recent years been presented as a new general-purpose technology that could underlie many aspects of social and economic life, including civics and urban governance. In an urban context, over the past few years, a number of actors have started to explore the application of distributed ledgers in amongst others smart city services as well as in blockchain for good and urban commons-projects. DLTs could become the administrative backbones of such projects, as the technology can be set-up as an administration, management and allocation tool for urban resources. With the addition of smart contracts, DLTs can further automate the processing of data and execution of decisions in urban resource management through algorithmic governance. This means that the technological set-up and design of such DLT based systems could have large implications for the ways urban resources are governed. Positive contributions are expected to be made toward (local) democracy, transparent governance, decentralization, and citizen empowerment. We argue that to fully scrutinize the implications for urban governance, a critical analysis of distributed ledger technologies is necessary. In this contribution, we explore the lens of "the city as a license" for such a critical analysis. Through this lens, the city is framed as a "rights-management-system," operated through DLT technology. Building upon Lefebvrian a right to the city-discourses, such an approach allows to ask important questions about the implications of DLTs for the democratic governance of cities in an open, inclusive urban culture. Through a technological exploration combined with a speculative approach, and guided by our interest in the rights management and agency that blockchains have been claimed to provide to their users, we trace six important issues: quantification; blockchain as a normative apparatus; the complicated relationship between transparency and accountability; the centralizing forces that act on blockchains; the degrees to which algorithmic rules can embed democratic law-making and enforcing; and finally, the limits of blockchain's trustlessness.

Keywords: blockchain, urban governance, distributed ledgers, platformization, public values 


\section{INTRODUCTION}

Recently, discussions have emerged around the application of blockchain in the domains of civics, urban governance and urban management. These discussions signal a shift in the understanding of distributed ledger technologies. Originally, they were understood mainly as financial instruments, with discussions focusing on the economic opportunities around digital currencies such as bitcoin and venture capital funded corporate blockchain startups. Gradually however, distributed ledgers such as blockchain have started to be seen as a new general-purpose technology that could underlie many aspects of social and economic life (Lipsey et al., 2005; Davidson et al., 2016; Kane, 2017), with important implications for urban governance.

In civil society, this shift is exemplified by various "blockchain for good" projects that have sprung up in recent years. A number of authors have also expressed the aptitude of blockchain to become the administrative backbone of civic economy, peer-topeer and urban commons projects (Pitt and Diaconescu, 2014; Bollier, 2015; Pazaitis et al., 2017; Antoniadis and Martignoni, 2018; Rozas et al., 2018; Bauwens and Pazaitis, 2019). The public sector has equally high expectations for the technology. At the launch of the European Blockchain Partnership, the European Commissioner for Digital Economy and Society Mariya Gabriel even claimed that "[I]n the future, all public services will use blockchain technology." In 2018, the European Commission also started the EU Blockchain Observatory and Forum to support such developments. Finally, the tech industry also sees a lot of opportunity to implement blockchain as a new infrastructure for various smart city technologies, providing a decentralized database and transaction infrastructure to keep track of services as varied as ad-hoc vehicle networks for the organization of autonomous cars and other forms of transport to energy communities (Sun et al., 2016; Shen and Pena-Mora, 2018; Bhushan et al., 2020).

What these approaches have in common is that they understand distributed ledgers and their smart contracts as part of emerging urban assemblages of technologies and practices (Farias and Bender, 2010), that allow for new ways of accounting and rights management. In these assemblages, distributed ledgers are well-placed to play a fundamental role in registering resource production, usage and transactions; keep track of account balances; and manage identities and rights. Moreover, with the addition of so-called smart contracts, distributed ledgers are set to play a role in the automated processing of data and conditional execution of transactions through algorithmic governance. Moving away from a main focus on "big data," these proposed services take the automated, conditional microtransactions between peers as a key element in their design. One way to describe this perspective is 'the city as a service, as introduced by Hwang (2009). Through this lens, the city is understood as a broad range of infrastructural services in the domains of mobility, leisure, entertainment, energy provision or health. DLTs are then seen as the administrative backbone that

${ }^{1}$ https://ec.europa.eu/digital-single-market/en/news/european-countries-joinblockchain-partnership allows for the easy micro-transactions, resource allocation, and rights management that underly these services to run smoothly and efficient.

However, as Kostakis and Bauwens have pointed out (Kostakis and Bauwens, 2014; Bauwens and Pazaitis, 2019), the integration of DLTs in the management of urban infrastructures is not merely a matter of making them more efficient or automatically contributing to a higher quality of life. Instead, they point out that the new types of accounting and transactions that DLTs afford, could lead to various outcomes. They could reinforce existing power structures, contribute to strengthening the logic of the market and centralizing power into the hands of a few recently emerged and centrally placed platform operators, or alternatively open up a direction toward different modes of social and economic organization such as the commons.

This article aims to contribute to the discussions on what the uptake of DLTs could mean for urban governance. We will approach this question through means of a technological exploration (Friedman et al., 2008). To what extent do the technological affordances of DLTs line up with desired societal outcomes projected onto the application of blockchains, and what could that imply for urban governance? This question is especially relevant in relation to the expectation that blockchain could contribute positively to civic self-organization and the management and governance of civic, commons-based peerto-peer economies, as more just and equitable modes of organization for society. To what extent do the technological affordances of blockchain line up with these expectations, or can we foresee value tensions that need to be resolved, between the technological set up of these systems and their desired outcomes?

To explore these issues, we propose an alternative and more critical lens to investigate the societal implications of DLTs: The city as a license. This perspective allows us to explore the introduction of distributed ledgers from a rights-to-thecity perspective and brings out questions that critically relate technological systems of governance to public values. Where the adage of The city as a service puts the focus on the usability and effectiveness of service provisions, The city as a license brings out questions in relation to power, inclusion and justice. It allows for laying a direct relation between technological developments and broader discussions about urban culture. The city as a license invites us to look critically at the city as a "rights-management-system," where the rights to use or contribute to particular resources are now organized through systems of algorithmic governance, taking shape through smart contracts and decentralized databases accounting and managing resources, rights and transactions. How does this relate to the ideal of the modern city as an open society built around inclusive public spaces and democratic governance (e.g., Sennett, 2006, 2018)

Following Foth (2017) call to the research community for exploring possible futures of blockchain technologies and their societal implications, we will explore these questions through a critical and speculative approach. We will depart from our own technological exploration of DLTs, including various speculative research projects and workshops, and connect these with broader 
debates around the technology (Nissen et al., 2017, 2018; Elsden et al., 2019a,b; Reshef Kera et al., 2019).

Our findings are presented as follows. In section Distributed Ledgers as a General-Purpose Technology, Part of SocioTechnological Assemblages we will contextualize the emergence of distributed ledgers as part of a broader assemblage of technologies, linking it to broader discussions on smart cities and platformization. We will move on to single out algorithmic governance as a key principle of these assemblages. The city as a license is then introduced as a lens through which the implications of DLTs can be discussed in connection to broader historical discussions about the right to the city and modern urban culture. In section Distributed Ledgers, Public Values and the Social Good we will turn to the affordances of blockchain that are thought to contribute positively to establishing democratic rights to the city and a more just and inclusive urban culture, as exemplified by various blockchain-for-good projects launched in the past few years. In section The City as a License: Six Traits of Distributed Ledgers That Need to be Taken Into Account we will compare these expectations and their underlying assumptions with a more in-depth technological exploration of DLTs. Through this we aim to highlight a number of value tensions that need to be resolved or taken into account when designing "DLTs for Good" from a right-to-the-city perspective.

\section{DISTRIBUTED LEDGERS AS A GENERAL-PURPOSE TECHNOLOGY, PART OF SOCIO-TECHNOLOGICAL ASSEMBLAGES}

\section{Platformization and Urban Governance by Platform}

The implications of distributed ledgers for urban governance could best be understood as one aspect of larger sociotechnological assemblages (Farias and Bender, 2010) that have been described under the rubric of platformization. Recently, a number of studies have pointed to the rise of digital platforms as new models for governance. The main argument is that platforms such as Uber, Airbnb, Facebook, and Amazon are not neutral, digitally enabled remediations of traditional marketplaces and communication channels. Rather they bring out a new logic and, according to some, should be understood as a new type of institution, imprinting their underlying logic on society at large. Srnicek (2017) has shown how the currently dominant platforms can be understood in the context of a shift in capitalism from the production of goods to the analysis of data, leading to a new hegemonic model of smart cities, disruptive businesses and a flexible workforce, dominated by large monopolistic firms. Others have pointed out that platform technologies also have the affordance to stimulate commons-based peer-production. Widening the scope beyond economics, Van Dijck et al. (2018) speak of a nascent platform society in which a small set of infrastructural platforms emerge as the central operators of a new societal infrastructure enabling sectoral platforms to re-organize societal sectors such as health, urban transport, education and journalism. In this line of thought, Gillespie (2017) speaks of "governance by platform" [italics ours]. Discussing social media platforms he states, "Both in terms of their impact on public discourse, and for the lived experience of its users, the rules these platforms impose themselves probably matter more than the legal restrictions under which they function." (Gillespie, 2017). van der Graaf (2018) applies this reasoning to an urban context, proposing to use the metaphor of the platform as a way to analyze and discuss shifts in the governance of cities, speaking of a platform urbanism.

In these instances, in an urban context, the term platform is used as a pars pro toto of an assemblage of various technologies and actors. Such a "stack" (Bratton, 2016) of recently developed digital technologies include sensor networks and Internet of Things-technologies that can actuate urban resources and measure their usage; and algorithmically governed online platforms that act as market places, connecting supply and demand in various domains.

In the context of urban governance, smart city-technologies can be understood as an example of such platformization (Kitchin, 2014; Cowley et al., 2017; De Waal and Dignum, 2017). Although definitions of smart cities differ widely (De Waal and Dignum, 2017), a trait that the many definitions hold in common is that all kinds of urban services can be optimized through the applications of various types of data that are assembled and processed in the city in real-time. The other way around, digital platforms also provide means for citizens to offer their own services as micro-entrepreneurs, or organize themselves around the management of resources in commonsbased local economies. For instance, through the systematic collection of (real-time) data, urban services such as transport can be optimized. Integrating algorithmic selection, gps-based location data, reputation systems, and electronic payments into a single user experience such as a taxi-app, offers urban consumers a smooth ride. At the same time, others can offer their services as taxi-drivers. An often-used metaphor is that of the control room (De Waal and Dignum, 2017), in which all kinds of urban processes can be monitored and managed in real-time, both from a top down perspective, as well as from the perspective of the user. His or her mobile phone becomes a personalized control-room from which a citizen can manage their own urban operations. Such "city as a service" (Hwang, 2009) approaches potentially form a powerful set of tools that provide new ways to manage and govern urban resources. How exactly this power is distributed differs greatly according to the design of a platform and the economics at play within it. For example, degrees of ownership, access, and participation might be influenced by these design choices.

\section{Blockchain Characteristics}

DLTs are a relatively new addition to this emerging "urban stack" as well as to broader visions of platformization. Decentralized database technologies such as blockchains are expected to play a role as an administrative layer in various platformized systems for resource management. Through these databases, actors can keep track of resources, identities, rights and account balances.

Blockchain is the most prominent current example of DLTs. As a technology it is hard to define, as it is sprawling into 
different domains with updated characteristics continuously. This means there is no catch-all definition as of yet. However, the blockchain is commonly described along a few main elements (Kewell et al., 2017; Wright and De Filippi, 2018, 431-32; Ølnes et al., 2017, 356; Elsden et al., 2018, 1; Rozas et al., 2018; Sultan et al., 2018) that can be summarized as follows: it is a distributed and decentralized network in which a verifiable record of a current (and historical) state of affairs is securely shared among the nodes. Generally, a consensus algorithm works to verify any new additions to the ledger, overcoming the need for human oversight and operating in what is called a trustless manner. These characteristics combined mean that blockchains are considered immutable. Sultan et al. further explain that these characteristics exist to different degrees according to the public or private nature of a blockchain (Sultan et al., 2018, 53). Whereas, first instances of blockchain functioned as decentralized ledgers, newer incarnations include so-called smart contracts. These are algorithmically encoded rules that can automate certain transactions based on pre-set conditions. This means that both performance and enforcement of these rules can be executed automatically, without the need (or possibility) for human interference (Wright and De Filippi, 2018; Cila et al., 2020). With these smart contracts embedded in its code, blockchain run systems can become Decentralized Autonomous Organizations (DAO), which were hailed around 2015 as a new way to build "transparent, efficient, fair, and democratic" organizations (DuPont, 2017).

\section{Algorithmic Regulation and the City as a License}

From the perspective of urban governance, the rise of distributed ledgers and their smart contracts may pertain an important shift. Distributed ledgers and their smart contracts can then be understood as part of what Yeung (2018) has described as 'the rise of automated data-driven systems to inform decisionmaking and regulate behavior', and perform what has been called 'algorithmic governance'. DTLs are then not just an accounting tool that keeps track of resources, they also govern the system and its users through its encoded rule sets. Yeung (2018) has described such algorithmic governance as:

\footnotetext{
"decision-making systems that regulate a domain of activity in order to manage risk or alter behavior through continual computational generation of knowledge from data emitted and directly collected (in real time on a continuous basis) from numerous dynamic components pertaining to the regulated environment in order to identify and, if necessary, automatically refine (or prompt refinement of) the system's operations to attain a pre-specified goal (Yeung, 2018).”
}

Such a perspective means that the introduction of distributed ledgers as a general-purpose technology for accounting and rights management may contribute to what De Filippi and Wright (2018) have described as, "a structural shift of power from legal rules and regulations administered by government authorities to code-based rules and protocols governed by decentralized blockchain-based networks." Various actors may encode particular rules and conditions of particular blockchain set-ups, de facto regulating various domains of urban society, with the actuation and enforcement of those rules delegated to Distributed Autonomous Organizations. According to Wright and De Filippi (2018), this means that increasingly we are subjected to what they call the "rule of code".

As an outcome of a speculative design workshop-in which the authors of this chapter participated-on a future in which such a rule of code has been realized, Elsden et al. (2019b) introduce the concept The city as a license as a lens to understand and explore what such a shift may entail. The city as a license, is described as "a future in which (semi-)autonomous digital systems administer rights and access to a broad variety of urban resources." For instance, with blockchain-based DAOs, parking places could be programmed to autonomously run a parking service, encoding particular rights and priorities in smart contracts with regard to whom is entitled to use a parking space under what conditions. The city as a whole could then be understood as "a series of licenses to make use of or contribute to the production of particular services such as parking, health, housing, energy or schooling" (Elsden et al., 2019b). It is important to note that this lens was introduced as a heuristic to critically explore the impact of DLTs, not as a design objective in itself. What does it mean if the city is increasingly governed through algorithmic systems that give out "licenses" to make use of urban resources? What are the underlying rule sets, and who has the power to determine and alter them? What room is left for interpretation, negotiation and exceptions when rules are encoded in software? And what new governmentalities does such a system produce?

If indeed new assemblages of technologies presented as urban services are introduced in such a manner to manage and govern urban resources, then the design of DLTs and their smart contracts become key in the ways that access and use of these resources is organized. Following again Yeung (2018), it becomes imperative to better understand exactly what kind of data is assembled and taken into account, and who has set the pre-specified goals. To vary on Gillespie (2017), in such a development, governance by DLT becomes a default condition in which urban resources, infrastructures and public spaces are managed. From that point of view, the city as a license can be understood as an alternative for the popular paradigm of the city as a service. Whereas, the latter would focus on the experience of consumers receiving individualized parking on demand services, 'the city as a license' directs attention to the politics of such systems. What world view, privileges and other political decisions are encoded in these systems? What are the conditions set, and who will be granted the privileges to make use of these services? Who (or what) makes these decisions, how is this made transparent and how can actors be held accountable?

\section{The City as a License and Discussions on Urban Culture}

The lens of the city as a license invites us to look at the city as a rights management system that becomes increasingly 
managed and formalized through DLTs and smart contract driven assemblages. Such a perspective allows a connection to be made between the discussion on DLTs and urban governance and debates about urban culture. In a broader approach of urban culture at large, various critics have made a plea for cities as open systems. It is the density, diversity, and ambiguity of cities that makes them places of inspiration and innovation, that allows citizens to build-up trust between one another, and that gives citizens agency to appropriate the various resources at offer from their own vantage points. As one of the most well-known proponents of this vision, throughout his career Richard Sennett has pleaded for urban design that is underspecified, that allows for a certain disorder and unresolved narratives (Sennett, 1970, 2006, 2018). According to Sennett, modernist, technocratic planning and neoliberal urban development with their focus on efficiency, functionality and profit have undermined this openness. New technologies such as those of smart cities and DLTs may further take away the agency of citizens and close off the path toward openness. To look at the city as a series of license, allows to critically investigate to what extent technological systems still allow for these ambiguities. To what extent do the licenses in DLTs demand that informal social and economic relations become formalized, in set relationships, roles and expectations? We will come back to this in section The City as a License: Six Traits of Distributed Ledgers That Need to be Taken Into Account.

More specifically, the City as license connects debates about DLT design and power structures with broader debates about the right to the city that was instigated by French philosopher Lefebvre in the 1960s. These rights as formulated by Lefebvre would consist of both access to resources as well as agency in the planning of future developments (Lefebvre et al., 1996). In the last few years, in the debate of smart cities, various critics have built upon Lefebvre to point to structural inequalities in the implementation of new urban technologies. Kitchin et al. invoke Lefebvre to "reframe, reimagine and remake the smart city as an emancipatory and empowering project; one that works for the benefit of all citizens and not just selected populations." (Kitchin et al., 2018; Cardullo et al., 2019). Similarly, Foth et al. (2015) want to "critique the established hegemony of the engineering and technologycentric epistemology embedded in any one proprietary smart city vision."

A perspective of "the city as a license" focusing on rights also matches with a broader general discourse emerging around platformization and smart cities. In their book The Platform Society, Van Dijck et al. analyze the rise of public platforms from a public values perspective and enquire how traditional arrangements for the safeguarding of these values are undermined or substituted by the mechanisms operative in digital platforms (Van Dijck et al.). Likewise, in the Netherlands The Rathenau Institute has called for better protection of public interests in the platform-based sharing and gig economies (Frenken et al., 2017).

\section{DISTRIBUTED LEDGERS, PUBLIC VALUES AND THE SOCIAL GOOD}

So far we have described DLTs and their smart contracts as a new layer in larger technological assemblages that are expected to play a role governing urban resource production, usage and allocation. And we have introduced the lens of the city as a license to analyze such a development from a perspective of citizen's rights and urban culture as an open system. Whereas, critics have pointed out that current smart city approaches in many cases undermine this right to the city, a more positive discourse has sprung up around the emergence of DLTs. As DLTs are inherently decentralized they may shift power back to citizens in their operation, for instance when applied in urban commons. Other characteristics may contribute to more transparent and accountable forms of urban governance. In this section we will further explore the characteristics of DLTs that are thought to contribute positively to society.

With regard to blockchain and distributed ledgers, various studies have explored how this technology could be applied for social good and provide opportunities for citizen empowerment. The Center for Social Innovation of the Graduate School of Stanford Business made a useful, albeit overly optimistic, analysis of 193 initiatives that aim to operationalize the blockchain for social good in the categories of: health; financial inclusion; energy, climate, and environment; philanthropy, aid, and donors; democracy and governance; agriculture; and land rights (Galen et al., 2018, 3). Bartoletti et al. broadly share these categories in their analysis of 120 "blockchain-enabled social good projects," adding digital identity, education, and human rights (Bartoletti et al., 2018 38).

More specifically, in the domain of urban resource management, DLTs are seen as technology that could further promote urban commons as systems of resource production, management, and governance within urban communities that focus on use value rather than exchange value, and are thought to contribute to more inclusive, sustainable urban societies, that are also more democratically governed by the commoners themselves. For instance, Rozas et al. (2018) see opportunities to deploy the blockchain in commons-projects to bring out a shift from "a culture of competition" to "a culture of cooperation." At least in theory, Rozas et al. conclude, blockchains have many affordances that match well with Elinor Ostrom's principles for successful commons. These decentralized technologies, they state, "could facilitate coordination, help to scale up commons governance or even be useful to share agreements and different forms of value amongst various communities in interoperable ways" (Rozas et al., 2018). Bauwens and Pazaitis similarly point out that the new affordances of blockchains are well-positioned to create new modes of accounting that have the potential to reward generative practices rather than extractive ones (Bauwens and Pazaitis, 2019).

Anticipation about this potential of the blockchain is usually based on one or more of four assumptions about the affordances of the blockchain. First, blockchains are expected to bring about more secure and trusted (transactional) systems because of their 
consensus algorithm and distributed, transparent nature. The Dutch Blockchain Coalition understands that the technology "due to its transparency and non-repudiation contributes to fundamental trust in our societal infrastructure" (Blockchain for Good: The Vision Mission of the Dutch Blockchain Coalition, 2018). The top benefit of the blockchain for social good cited in the Center for Social Innovation's report is the technology's contribution to the "reduction of risk/fraud or increase in integrity/transparency” (Galen et al., 2018, 4).

Second, blockchains are perceived as democratizing technology because of their reliance on consensus algorithms instead of opaque central authorities (Ølnes et al., 2017, 363). While central authorities may be influenced by politics or corporate interests, decisions based on algorithmic calculations are presumed to be more objective or neutral (Galen et al., 2018 , 12). Using blockchains in systems that are meant to support democratic processes are seen to be a tool for citizens to hold governments accountable (Galen et al., 2018, 21). By making supply chains transparent, blockchains are also assumed to facilitate fair payment for those vulnerable to exploitation located at the often opaque start of the chain (e.g., farmers of coffee beans in the Global South) (Galen et al. 16). Another use case that is mentioned is the recording of land and property rights on the blockchain, especially in countries where such registries are not well-established or considered trustworthy (Pisa and Juden, 2017, 28)

Third, the decentralized and transparent nature of the blockchain is understood to give people more control and agency over, for example, their digital identities or the set of rules that are encoded in their DLTs. Individuals can determine on a case-bycase basis which pieces of personal information are made visible to whom (Kewell et al., 2017, 434; Galen et al., 2018, 27). Some initiatives use the blockchain to provide digital identities (and concordantly control over them) to those who, due to geopolitical or economic reasons, have been left out of these infrastructures traditionally, for example the unbanked (Pisa and Juden, 2017, 25-26; Galen et al., 2018, 3, 29, 40). In another set of examples of democratization, authors point to the ability for commoners to design their own systems and its encoded values. In commonsbased peer production projects, tokenization also gives members of a community the power to denote what kind of activities they deem of value. Care giving and other forms of emotional value that in traditional economic systems have gone by unawarded, can now be counted as contributions to a common good. This "provides an opportunity to rethink existing power dynamics within CBPP communities” (Rozas et al., 2018). Bauwens and Pazaitis (2019) speak of protocol coops as a governance form in which leagues of cities could set up alternative infrastructures that allow for the rewarding of generative contributions to the economy.

Fourth, relying on quantification for its algorithm, blockchain makes efficient and minute bookkeeping possible in domains in which it has historically been difficult to do so, for example the conditional distribution of charity, international aid funds distribution, or the tracking of the impact of philanthropic donations (Galen et al., 2018, 60-61; Pisa and Juden, 2017, 31). Not unimportantly, the use of blockchain for the international transfer of money can reduce overhead costs (Pisa and Juden, 2017, 19-20; Galen et al., 2018, 61). Another case is made in relation to education, in which blockchain-based accreditation systems could provide more individualized, diversified, and detailed proof of particular comprehension or skills, improving existing homogenizing educational structures (Galen et al., 2018 69).

Taken together, looking at it through the lens of the city as a license, blockchain has the affordances to have a positive outcome for the management of public values and public and collective interests. It provides a tool for local communities to organize their own resources, rights and identity management. Who is entitled to what right to a particular resource can be organized in a bottom-up fashion and the technology itself is expected to make these processes more transparent and accountable. Most authors in this space note that these affordances will not automatically bring out such a scenario, and are aware of the risks involved that DLTs will rather further centralize power and undermine agency for citizens. It is therefore important that we take a deeper look at the underlying affordances of blockchain, and enquire to what extent distributed ledgers may or may not contribute to a fairly, democratically governed city.

\section{THE CITY AS A LICENSE: SIX TRAITS OF DISTRIBUTED LEDGERS THAT NEED TO BE TAKEN INTO ACCOUNT}

When exploring the technological affordances of new technologies, it is imperative that we go beyond the functionalities and procedures they enable. Mediating sociality, and formulating the institutional in radically different ways than before, can be understood as constructing "a new social model" (Bauwens et al. 8). When applied in such a way, new technologies are embedded in, and have an influence on, cultures of use, (in)equalities, democratic participation, power dynamics, and social cohesion. We will now shift our analysis from the procedural analysis that is often used to underwrite the "blockchain for good" claims, to a further analysis that seeks to explore the aspects of social life that may be affected by the introduction of distributed ledgers. Guided by our "city as a license" interest in the rights management and agency that blockchains are hoped to provide to their users, we will trace six important issues: quantification; blockchain as a normative apparatus; the complicated relationship between transparency and accountability; the centralizing forces that act on blockchains; the degrees to which algorithmic rules can embed democratic law-making and enforcing; and finally, the limits of blockchain's trustlessness.

\section{Quantification}

A first important issue is the affordance of distributed ledgers to turn any kind of asset, resource use or social relation into a quantifiable unit. From "a city as a license" -perspective, this is exactly what allows DAOs to give or refuse users conditional access to a particular asset or relationship, based for instance on their reputation, profile, status, account balances, prior usage, 
subscriptions, etcetera that are stored in quantified units on the distributed ledger. To stick with our parking example: a parking place could rent itself out when it can (a) measure the usage of the parking place (in minutes or square meters occupied); (b) recognize the identity of a vehicle or person seeking access; (c) connect this identity with a database that contains particular rights and privileges (e.g., priority or reduced rates for doctors or people who have been carpooling, or perhaps a ban for a car with a particular license plate due to prior traffic violations), (d) tie this is in with external information (e.g., traffic conditions, event happening nearby that may spur an increase in demand) to adjust pricing to expected demand, and (e) connect this with a transaction system that allows for payment in any kind of token.

While such a combination of datafication and algorithmic regulation may work for parking, it becomes more problematic when public values and informal social relationships are at stake, as these are not easily converted into measurable units. And if they are, the risk is that these objectified quantifications obscure their underlying values and dynamics with citizens internalizing the logic of the quantified system, rather than subscribing to these underlying values.

To explain this with an example, it is important to understand that reduced to its very basics, blockchain is an administration technology. It is a way to keep track of assets on a ledger. These assets can be anything from currencies to property, traces of provenance, or access rights. In order to create a single unit of account, these assets are represented by tokens on the blockchain, similar to the way money functions as an intermediate between different forms of value. By translating assets into tokens, they are quantified (if they are not already expressed in quantities to begin with). A community aiming to reduce their collective energy use through sharing resources (assets) like an electric car, energy generated from collectively owned solar panels, and a community kitchen could use a blockchain system to keep track of the various uses and contributions of community members. Representing their assets by abstracted quantities of tokens on the blockchain, this community would have to agree on an exchange rate between one cooking session, an hour-long car trip, and one kilowatt hour of energy. The community might also want to reward certain tasks with tokens, like cleaning and upkeep of the system, which will also have to fit into this exchange rate. Based on how much a community member contributes, the blockchain could then automatically distribute their rightful share (a temporary license to make use of a particular resource) of the community's solar energy or unlock access to the shared electric car. As Rozas et al. (2018) has pointed out, this option for communities to selfdetermine which contributions can be valued, is potentially an empowering force. Yet, continues Rozas, it is not without risks, as it could lead to data-fetishism and extreme quantification (Rozas et al., 2018).

To understand what such a total datafication could entail, it is instructive to look at existing examples of quantification outside of blockchain applications. Quantification and the counting of social interactions have a longer history and have been analyzed for their effects in different contexts of application (e.g., Barsh, 1993; Andreas, 2008; Espeland and Stevens, 2008;
Davis et al., 2012a,b; Scott, 1998; Hacking, 1999; Bowker and Star, 2000). Sally Engle Merry conceptualizes the phenomenon of creating indicators for social realities through quantification as a technology of knowledge. She writes "[w]hile we assume that [indicators] describe the world, they actually construct that world" (Engle Merry, 2016, 33). Developers of systems of quantified representation might be aware of the compromises and choices they made in order to represent a messy social life through categories and numbers, but these decisions are not visible to others. What is represented, gets "stripped of their context, history, and meaning" (Engle Merry 1) in this process. Quantified knowledge, intended or not, carries with it an appearance of objectivity, efficiency, and consistency and is readily operationalized for decision-making and governance as a result (Davis et al., 2012a, 84; Engle Merry, 2016, 209), especially in the case of automated, algorithmic processes.

In his book The Tyranny of Metrics Jerry Z. Muller (2018) analyzes what such quantification has meant for sectors as diverse as the military, finance, medicine, and education. It is these latter two that provide useful insight with regards to the use of metrics in domains that are understood to exist at least in part outside of the market. Muller describes how during the austerityridden 1980s, in an international rush of Foucauldian neoliberal governmentality, politicians and policymakers increasingly steered these non-profit domains toward business-like strategies in order to save money. Performance indicators were developed for universities and hospitals. These quantified performances were then tied to monetary rewards and punishments, and made visible to the broader public so that competition between institutions would occur. Having more information was thought to allow citizens to make informed consumer decisions, ultimately driving prices down and quality up. While in business performance is somewhat straightforwardly assessed in terms of financial gain, organizations that have a central societal purpose often have more diverse and diffuse goals that are more difficult to measure in metrics (Muller, 2018, 43-44, 51-53). Holding organizations aiming for a social good accountable according to only that which is quantitatively measurable therefore misses their raison d'être at least to some degree and potentially frames their users as consumers of marketed products instead of as citizens with rights.

Although far removed from the aim for social good, Muller's account of the "short-termism" at work in financial markets in the years before the crisis of 2008 is useful for our purpose also. Quarterly earnings are a main influence on a company's performance on the stock market, and thus are made of central importance in management strategies, linking bonuses or commissions to favorable quarterly reports. This subsequently leads team managers and individual employees to focus their work on achieving goals that can be reached within 3 months, often at the expense of long term investments like employee education or maintenance (Muller 147-50). By measuring effects immediately and making them visible (transparent), quantification can lead to decisions that favor the short term over the long term, an effect that is amplified by the necessary simplification of social reality as it gets made legible in abstracted indicators (Scott, 1998, 19-21). Presenting continuous quantified 
insights invites continuous action upon that which is visible in the measurement, and only that.

Thus, by creating countable representations of contributions and participation in a community, social relations are made explicit, formalized, and are standardized where before they were implicit, personal, and flexible. Hierarchies representing who contributes to or uses most can be made in which the distinction between the things that are countable and counted, and the things that are not become consequential. While buying groceries for your elderly neighbor might add to your reputation in a community normally, if it is not counted by the neighborhood system, it will not be visible in its hierarchies.

The short-termism Muller describes does more than just disincentivize necessary but less instantly rewarding tasks like maintenance. In the same move, it also discourages innovation and creativity. Using metrics in effect predetermines which kinds of efforts are rewarded and which are not. If the city is indeed understood as a collection of licenses that give conditional access to resources, based on prior engagements, reputations and other quantified indicators, the risk is that people will conform their participation to these already existing categories in order to be legible to and deemed valuable by the system. This makes other types of activities, however socially relevant they might be, less valuable and therefore less favorable. This will ultimately lead to a situation in which innovation and creativity are disincentivized, because they stray from the pre-defined categories of value (Muller, 2018, 20, 171).

To conclude, blockchains favor things that can be quantified. This tendency stems from the ideological beginnings of the technology. Designed as a way to route around corruptible centralized authority and to facilitate globalized trust through cryptography, it has always relied on the countable and follows the logic of objectivity through quantification. As a result, the influence of human subjectivity and the social have always been minimized in blockchain technology (Terranova and Fumagalli, 2015 , 154). However, the centrality of the quantification, as perceived as objective, may actually backfire when such a system is introduced in social domains, or in systems that are to manage licenses in relation to public values. As the work of Muller, Scott, and Engle Merry has shown, this emphasis on quantified information can lead to market logics, short-termism, simplification of complex social systems, and unaccountable forms of power. These are worrying trends in relation to the aim for blockchain systems to support social good.

\section{The Blockchain as a Normative Apparatus}

Quantification as described above is not just an act of translation of one type of value into another. Seen through the "city as a license" -lens it also becomes an important apparatus to apply particular normative frameworks toward a social and/or economic system. As we have shown above, numbers themselves acquire the agency to shape what they measure when they are deemed important. By linking performance indicators to monetary rewards or other tokens registered on the blockchain, the numbers that influence these outcomes are made to matter. For instance, in an imagined blockchain future, access to a city park (a license to use the park) can be based on an individual's participation in plastic recycling schemes. Thus, numbers that matter have the power to socialize their subjects, to lightly nudge people to conform to norms for example by rewarding good behavior. Theodore M. Porter described numbers as "among the gentlest and yet most pervasive forms of power in modern democracies" (Porter, 1995, 45).

The relation between quantification and power is investigated further by Jacqueline Wernimont, who analyzes historical examples of systems that quantify human behavior to reveal their congruence with established power relations (Wernimont, 2018, 3). Blockchains can be seen as new instances of what Wernimont calls quantum media, which

\footnotetext{
"refract human behavior and bodily action as a stream of numbers. It is a highly lossy remediation that abstracts action in the world into quanta, and is situated in a late moment in liberal thought dominated by notions of personal power and agency." (Wernimont, 2018, 162)
}

This belief in personal agency through numbers persists today, as the popularity of self-quantification hrough productivity tracker apps or devices like the Fitbit shows. These technologies overrule inherent knowledge of the self one might think to have by claiming that the truth is to be found in the abstracted data. "[S]elf-tracking can quickly be leveraged by others to know those who aren't trusted to know themselves" (Wernimont, 2018, 157). It produces a way of knowing the subject that is mediated by categories and indicators that are determined by the interests of those that govern the systems used. Moreover, in blackboxed and often proprietary systems, not everyone has the same access and agency to act on the way they are made visible in them. These differences unsurprisingly follow pre-exiting societal divides, like income, race, and gender (Davis et al., 2012b, 81; Crawford et al., 2015; Wernimont, 2018, 159, 162).

Following Ananny and Crawford (2018) and Gillespie (2014) in their understandings of technologies as inextricably entangled with human actors and practices, this could produce the existence of multiple data cultures: categories have different meanings for different people, and data practices vary between locations and communities. Looking at data use in cities, Jo Bates analyzes data cultures in relation to power. She emphasizes the need to think about how data systems are structured according to existing power relations, how they are potentially differently understood and acted upon by participants, and tensions between these, respectively, top-down and bottom-up perspectives (Bates, 2018, 192). Different people relate differently to the fact that their social lives are represented in urban data systems. They might feel surveilled and try to resist, manipulate, or subvert forms of data capture. Those hoping to gain from the system might try to game it for their own benefit. Alternatively, people might not understand these systems, or feel apathetic toward them, both possibly leading to solely passive participation. Finally, different people have different abilities to participate, whether that be in terms of economic status or cognitive or physical characteristics. Bates describes how these struggles and acts of resistance have an impact on how a social reality is visible in a system of representation (Bates, 2018, 197). Whether someone is 
consciously disrupting it or not, a system just makes visible what it is told it can see, not what is actually there.

This inability of systems of representation to capture their underlying reality exactly can make them vulnerable to corruption and gaming, especially in settings where an idealistic mission is central (Muller, 2018, 19, 77-78, 121). This is expressed well in what is known as Campbell's Law:

The more any quantitative social indicator is used for social decision-making, the more subject it will be to corruption pressures and the more apt it will be to distort and corrupt the social processes it is intended to monitor. (Campbell, 1979, 85)

Those who have more agency to distort the system, or to pay to get exemptions from the rules, will be able to get more favorable results. In a city of blockchain-based traffic dispersal for the even distribution of polluting vehicles, we can imagine that a group of well-organized, wealthier neighbors could put political or economic pressure on the administration to make sure that their neighborhood is exempted from the route planning algorithms and therefore does not show up on the map that truckers use to navigate the city. Early examples of these kind of politics are the complaints that citizen in San Francisco have been filing against Waze for directing rush hour traffic through their residential neighborhoods.

The inclusion of normative frameworks in algorithmic regulation also opens up possibilities for a highly flexible and customizable application of rules. With smart contract, the rules encoded in the system can be made conditional of various types of datafied input, from traffic conditions and the weather forecast to user status and reputation systems. Rather than have one particular rule set for the city at large, access to streets, can be regulated through particular flexible right management schemes that for instance gives or declines the right for particular categories of traffic users to use or not use a particular street at a particular time.

For regulators, this opens up the possibility for microregulation. Blockchain solutions, aided by smart contracts and cryptocurrencies, likely aim for beneficial social outcomes in highly specific problem areas. To deal with overwhelming numbers of tourists, a city like Amsterdam might put in place tokens that are worth more in less crowded areas of the city, drawing tourists out of the congested city center (Elsden et al., 2019b), and perhaps release those conditions in offseason or off-hour periods. Locals on social welfare might at the same time receive their benefits in a specific token that limits their use to particular white-listed outlets or items, an update of current systems of welfare administration that surveil the poor disproportionately (Maréchal, 2018). In this way, a city can become host to a rich layering of different systems of value and accessibility, designed and maintained by a decentralized network of institutions, organizations, and communities. Localized value systems have the potential to better reflect local values and specificities.

Such a City as a license scenario may be the dream of some regulators. DAOs with rights management allow them to microregulate conditions, even in real time, creating a multiplicity of systems that together make up the city. The right to use certain streets may be revoked for particular types of transport during particular times, access to services can be restricted based on reputations and status, and taxes can be adjusted in real time to stipulate desired behaviors toward outcomes for the public good. However, at the same time, such a constellation of systems as a whole has the potential to become too complex and opaque to adequately and democratically govern. Whereas, the basic promise of distributed ledgers is one of transparency, such systems may actually result in the reverse and open up possibilities for new ways of wielding influence by third parties and even corruption. How would citizens or consumers know to what regimes they are subjected and why? And what does it mean for citizens' trust in institutions if the underlying rules can change from moment to moment?

\section{Transparency and Accountability in Distributed Algorithmic Networks}

Their distributed ledgers potentially allow blockchains to achieve new levels of transparency. These ledgers can make previously hidden exchanges or processes visible, and therefore offer new means to hold others accountable (Rozas et al., 2018). Especially for the advance of sustainable food production and fair fashion, logistics is a sector that receives particular attention of blockchain for good initiatives. Here, blockchains are explored for their potential to make supply chains more transparent and honest (Blockchain for Good: The Vision Mission of the Dutch Blockchain Coalition, 2018) by stamping particular certificates or licenses (e.g., fair trade) or other marks (proof of origin of a resource) on the blockchain. However, when a blockchain handles personal information, for example in an urban blockchain-based system that handles basic daily needs like transportation, transparency becomes more problematic. Rozas et al. (2018) are concerned with commons communities that share resources using blockchains, and point toward the privacyrelated issues, like the clash of a principle like the right to be forgotten with the immutability of blockchain ledgers. On the one hand, local systems that make contributions to the commons transparent could provide an overall trust in the system and undermine the opportunities for free riders. Yet, that would mean that individual members of the commons would have to give up a bit of their privacy by enabling their contributions to be made public. This could become more problematic in cases of algorithmic governance. For instance, if a car sharing system belonging to a local community has rules about priorities (e.g., medical emergencies), this means that members that want to exercise these privileges have to make their cause public to the system. As Cila et al. (2020) have demonstrated, such systems will inadvertently bring up a number of design dilemmas that designers have to consider, as to when transparency is desired, and to what extent infringes on privacy are justifiable.

On the level of the city, if cities become a mesh network of various right management systems organized through DLTs, some by project developers, others by urban commons, others by private entities exploiting toll roads, and others again by local governments, how can a citizen understand under which regime 
he currently falls, and what the rules are? How transparent will these various right management systems be interfaced, would all their rules be clear and understandable for citizens, and would citizens also have the right to opt-out from any of these regimes?

On the opposite end of the spectrum, dealing with global blockchain infrastructures brings out another problem. The technology does not have a headquarter location, its code is stored across a global network of users, and in cases where applications are co-produced by a network of open source code contributors there is no delineable origin. Because of these characteristics, even if there would be a way to hold a distributed network accountable, the question remains: to which laws? In this sense, DAOs can run their code without adhering to all or even any of the jurisdictions they have an effect in De Filippi and Hassan $(2016,3)$. This issue is reminiscent of the way platforms like Facebook or Airbnb operate above or beside local laws either by instituting their own additional rules (e.g., Facebook's nudity guidelines based on American sensitivities that at least in some European localities miss the mark) or denying their local responsibilities and consequences (e.g., Airbnb's refusal to cooperate with Amsterdam's authorities to enforce the city's limitations on the use of the platform). From the City as a license-perspective, that could be very problematic, as it means that local government may lose control over the actors that set the conditions for these licenses in the first place. While certain processes may become more transparent (often at the cost of privacy), it does not automatically lead to better accountability.

\section{Centralizing Forces}

One of the important claims in the discourse around blockchain for social good is that distributed ledgers can be organized in a decentralized manner and run without central authorities. From the city as a license-perspective, that would theoretically give local communities the possibility to set up their own systems and encode their own rules. Think of local energy communities defining their own economic principles or volunteer-run organizations that decide on non-monetary rewards. Whereas, this could certainly be attractive in certain cases, it is far from granted that such a decentralized structure will remain in place.

In that regard blockchain's development may show more historical similarities with that of the internet at large. Just like in the early nineties the internet was for a large part occupied by homepages made by individuals, but is now dominated by multinationals like Google and Facebook. Likewise, the bitcoin blockchain is not as distributed now as it once was (Bauwens et al., 2019, 38). For example, due to their own popularity, interfaces had to be designed to make cryptocurrencies accessible to users that are not tech-literate. The necessity of wallets and other interfaces centralizes blockchain usage into particular applications. The energy community or volunteerrun organization from above might fall back on standardized systems funneling data through centralized services. This is illustrated by the fact that currently only two wallets dominate the cryptocurrency space. ${ }^{2}$ On a more societal level, the prevailing

${ }^{2}$ https://blog.blockonomics.co/why-bitcoin-is-becoming-centralized$41 \mathrm{f} 62 \mathrm{cc} 15 \mathrm{e} 91$. economic logics of neoliberal capitalism don't spare blockchainbased systems from their monopolizing tendencies (Herian, 2018, 50). Mining power in the bitcoin network is dominated by just a few actors for example, ${ }^{3}$ making it increasingly vulnerable to $51 \%$ attacks which effectively overcomes the blockchain's immutability.

It is as yet unclear how a distributed network would concretely be implemented in a societal context. How much of a city blockchain would for example be distributed among the computers of user-citizens? If blockchains that manage rights in a city become dominant, would citizens still have options to use other routes to be granted their rights? And not insignificant: how much agency, control, and opacity is a government or organization actually willing to give up to such a system, even if they have the public good in mind? An extreme example can be found in Facebook's proposed blockchain-based currency Libra, officially announced in 2019. In its white paper, Libra is conceptualized expressly in term of its proposed social contribution, as a way to "empower billions of people," and having "the goal of building more inclusive financial options for the world" [An Introduction to Libra. (n.d.)]. At the same time, at least initially, the technology will neither be decentralized (the companies that back the currency also control the blockchain), nor permissionless (only certain actors contribute to the consensus algorithm, instead of the whole network). This means it is a stretch to call Libra blockchain-based in reality (O'Dwyer 2019). In a culture of socially responsible entrepreneurialism, blockchain terminology is vulnerable to cooption for corporate interests under the guise of dedication to "the social good".

\section{Code Is Law}

With 'the city as a License', the licensing process and its accompanying rule sets are encoded on the blockchain. This means that licenses to access or use urban services are automatically given out or revoked without human intervention. The blockchain represents at the same time the regulations as well as their enforcement. Such automated compliance may undermine the democratic right to contest rules (Yeung 2018). The Dutch Raad van State has also warned against the limited opportunities for interpretation of rules in such algorithmic regulation (van State, 2018).

These aspects of smart contracts and DAOs have led several researchers to re-investigate Lawrence Lessig's 'code is law' formulation. Lessig's work on the laws at work in cyberspace uncovered the ways in which the code of the internet regulates user's behavior. Lessig showed that computer code functions similar to natural laws. Instead of threatening punishment like the law does, nature, and code, function by making certain acts simply impossible: "[w]e live life subject to the code, as we live life subject to nature" (Lessig, 1997, 184). An important difference with nature is of course that code is written by fallible humans with individual biases. Code is also changeable and subject to politics. Because behavior outside of the limitations of code is

${ }^{3}$ https://blog.blockonomics.co/why-bitcoin-is-becoming-centralized$41 \mathrm{f} 62 \mathrm{cc} 15 \mathrm{e} 91$. 
impossible, this can make political world views into naturalized ranges of possibility (Lessig, 1997, 183-84).

Digital rights management technology like iTunes song copy protection software use the laws of code to enforce judicial copyright laws (at least for the not-so-technically-literate). As written laws are captured in algorithmic computer language, a degree of their ambiguity and flexibility is lost (De Filippi and Hassan, 2016, 10). It is quite impossible for example, to compose an algorithm that can determine a highly ambiguous and contextreliant exception to copyright law such as fair use. To cede the interpretation of ambiguous laws to formalized code often means to reduce the freedoms that the ambiguity of traditional law provides (De Filippi and Hassan, 2016, 7).

When laws, rules, or agreements are captured in smart contracts, they become unbreachable, automatically executed, and possibly tied to physical objects (Wright and De Filippi, 2018, 26). Once a DAO has been set in motion, they are in theory unstoppable (Wright and De Filippi, 2018, 17). Unless a kill-switch was explicitly designed, or a complete network is shut down, a DAO finishes its code and implements its consequences. In effect, the laws and rules coded into a smart contract (e.g., a speed limit on a road), are interpreted and made to have an effect algorithmically (e.g., sensors detect a car going over the speed limit and charge the owner of the car a fine), irrespective of context (e.g., medical emergency) or other mitigating circumstances (De Filippi and Hassan, 2016, 14).

Code is written in advance of real situations happening ("exante"), and so can only regulate for what can be expected. Once the code runs, edge-cases will be unforgivingly dealt with by an algorithm that keeps plowing on. The way the law is traditionally operationalized grants humans negotiating, intervening, and ultimate deciding powers, while smart contracts and DAOs generally do not. While algorithmic decision-making is fast, we must not lose the human democratic debate that is central to interpreting and implementing the law (De Filippi and Hassan, $2016,6,15,18)$. This could potentially be done by designing smart contracts that can be interrupted for human voting on set times, or in specific cases (Wright and De Filippi 50), which is called off-chain governance (Reijers et al., 2018, 3). How this should be done, especially in relation to edge-cases that are not recognized as such, is something to be tested.

Also at a city level, it is unclear to what extent various rules and rights will become formalized in DLT systems. Will the city indeed become a mesh network of various regimes of rights-management? Will local governments set up a metainfrastructure of encoded rules or design mandatory "platformplug ins," that various DLT-systems will be subjected to? On the one hand such systems may ensure public values to become safeguarded. At the same time, when all rules, social arrangements and forms of resource management are encoded into a City as a license-approach, this may undermine the functioning of the city as an open, ambiguous system in which new cultural, social and economic relations may emerge. Finally, this will also lead to questions with regard to inclusion. What about the informal settlers or the undocumented that still can make use of the services offered in today's cities, even if they do not have any formal rights. How would such populations gain access to the formal systems that may govern urban spaces?

\section{Trustless Trust Reconsidered}

A final theme we would like to address in relation to the application of distributed ledgers in urban systems, is the notion of trustless trust. In the technology community, the word trustlessness is used to indicate that there is no central authority that needs to be trusted, for example in terms of its benevolence and capability. Blockchain is often discussed as a technology that due to its architecture and validation procedures enables cryptographic proof: the rights, identities, transactions, and licenses managed can be trusted as valid without the need for a trusted intermediary.

This faith in the technology can be traced back to its origins. Blockchain technology was first described in Satoshi Nakamoto's white paper on bitcoin (Nakamoto 2008) amid the financial crisis of the late 00s. Kick-started by rampant subprime mortgages, the crisis featured collapsing banks and a recession that had not been experienced in a lifetime, leading to a widespread wish for alternative financial systems. Nakamoto's technology eliminated the need for central authorities in economic transfers, and found fertile ground at a time when mistrust of banks was at a high point in recent history (Sapienza and Zingales, 2012, 130). Nakamoto's bitcoin would, supported by its blockchain, replace banks and governments with a distributed and 'trustless' network, giving individuals autonomy over their money.

Blockchains theoretically achieve their trustlessness by randomly assigning the power to validate transactions across a network of non-hierarchical participants. In abstract, this does indeed overcome the need to trust a central authority but in the real application of these theoretical systems, there are caveats. As a case in point, cryptographer and legal scholar Nick Szabo prefers the term 'trust-minimized', stating that trustless is "exaggerated shorthand" (Szabo 2014). Jaya Klara Brekke provides an in-depth analysis of how the political is mediated through the blockchain, showing that blockchain systems are not neutral carriers of mathematical proof.

Licenses, after all, can only be given out by trusted and legitimate institutions. Outsourcing this process to the blockchain presupposes trust in the legality, validity, fairness, impartiality, and robustness of the code of that blockchain (Al Khalil et al., 2017, 515; Hawlitschek et al., 2018, 57). This becomes especially pertinent when dealing with a user-base that does not have the technological skills to investigate the inner workings of the system they are using (Hawlitschek et al., 2018, 60). When code is not readable by the average human being, participation comes down to trusting other people (e.g., programmers) to do their job according to standards and values you agree with (Ølnes et al., 2017, 363). Blockchain-based systems that are implemented in administrative systems that citizens rely on for basic needs require maximal care in terms of the legibility of their systems by diversely educated and technically-capable citizens. In the case of cryptocurrencies, we see a proliferation of wallets, or digital interfaces to manage blockchain-based coins. All of these wallets have their own terms of service, privacy statements, and business models, as well as vulnerabilities to hacks. Interfaces like these 
will need to be designed for any blockchain system to allow citizen-users to interact with the technology, and these need to be trusted by their users for them to be taken up (Hawlitschek et al., 2018, 51).

Taken together with our earlier concerns around the centralizing tendencies in blockchain systems, the patchwork of user agreements and third-party interests that potentially make up an urban blockchain assemblage endangers their trustlessness. Referring to blockchains as trustless runs the risk of concealing these very real threats to their trustworthiness.

\section{CONCLUSION AND DISCUSSION}

In this article we aimed to contribute to debates about the application of blockchain in the domain of urban governance. We have shown that distributed ledgers-of which blockchain is an example-can be understood as a new general-purpose technology, that could play an important role as an administrative layer and actuating agent in various assemblages of technologies and use practices. These systems allow for new models to monitor, manage and actuate all kinds of urban processes. Examples vary from smart city services such as the management of parking spaces to the organization of local, commons-based peer-to-peer economies.

We introduced "the city as a license" as a lens to explore these platforms or smart city services from a perspective of governance. As such we proposed to think of automated blockchain-based platforms as actors that give out licenses to temporary make use of resources, based on conditions encoded in smart-contracts through algorithmic governance. The city, seen through that lens, becomes a rights management system, or more likely a system of systems of rights management, and that perspective brings out questions in relation to power, agency, accountability and transparency.

In terms of civics and urban governance, there is a hope that such systems could contribute to fairer, democratic systems and empower citizens in various ways. This direction is currently explored in various "blockchain for good" processes as well as in movements around urban commons. While we value this approach, we also argue that to fully scrutinize the implications of distributed ledgers for urban governance, a more critical analysis of distributed ledger technologies is necessary.

We set out on that course through a technological exploration of distributed ledger technologies. In combination with a speculative approach, we thus explored a broad array of possible implications of distributed ledger technologies for urban governance. The themes brought up here as an outcome of that process are not conclusive nor exhaustive, but they do point to a number of important tensions that should be of concern to policy makers, designers of blockchain-based systems as well of citizens.

The use of blockchains in situations where social relations are at stake is risky, as informal social relations need to be quantified and therefore become part of formal economic systems. The transparency that could make these systems and the contributions of their users accountable, could at the same time undermine the privacy of its users. In addition, these formal systems can be seen as normative apparatuses that are highly likely to nudge citizen behavior. This may give regulators and system operators the opportunity to implement highly flexible micro-regulations, leading to a multiplicity of systems and value sets operative in the city. In turn this could make the governance of these systems highly opaque and open them up for untransparent influences by outside actors or even corruption. The automatic encoding and enforcement of rules can also become problematic, as such automated systems leave no room for interpretation of the rules and may be not be prepared to accurately deal with unforeseen situations at the time of encoding the rules. Whereas, blockchain-based ledgers are envisaged as empowering to citizens because of their decentralized character, and their architecture that can invoke "trustless trust," there is also a risk that these networks will become dominated by a few central actors again, not unlike the internet itself. How these actors and their code could be held accountable by local legislators is not directly clear. Likewise, the trust that citizens may have in these systems could be undermined by their multiplicities and opaque form of algorithmic governance.

All this is not to say that nothing good can be expected from distributed ledger technologies. On the contrary, we think that an approach to the blockchain that focuses on public values, citizens' rights and social good is highly desirable, as long as it situates these technologies as part of specific social, political, and economic realities. Much more research is needed to further explore the affordances of distributed ledgers in relation to urban governance, both from a perspective of technological explorations as well as from a design-perspective in which experiments with distributed ledgers can shine more light on the desirability of certain features. Yet, researchers and designers should not be naive, and we hope that the lens of the city as a license introduced here provides points of departure for further critical investigation of distributed ledger technologies for the public good and citizens' rights from a governance perspective.

\section{AUTHOR CONTRIBUTIONS}

IG and MD wrote a first draft. GF and NC contributed to this draft. All authors contributed to manuscript revision, read and approved the submitted version and contributed to the technological exploration, and literature review.

\section{FUNDING}

This paper was written as part of the Design Thinking for the Circular Economy research project. This research was cofinanced by Regieorgaan SIA, part of the Dutch Research Council (NWO).

\section{ACKNOWLEDGMENTS}

This manuscript was written as part of the SIA RAAK-MKB grant Design Thinking for the Circular Economy. The notion of the 
city as a license was first developed in the workshop Making the Blockchain Civic, co-organized by the authors of this papers as a collaboration between Amsterdam University of Applied Sciences's Faculty of Digital Media and Creative Industries, and Northumbria University's interdisciplinary NorTH Lab. We thank Chris Elsden, Anne Spaa, and John Vines for co-organizing

\section{REFERENCES}

“An Introduction to Libra." (n.d). Libra. Available online at: https://libra.org/enUS/white-paper/ (accessed December 19, 2019).

Al Khalil, F., Butler, T., O’Brien, L., and Ceci, M. (2017). “Trust in smart contracts is a process, as well," in Financial Cryptography and Data Security, eds M. Brenner, K. Rohloff, J. Bonneau, A. Miller, P. Y. A. Ryan, V. Teague, A. Bracciali, M. Sala, F. Pintore, and M. Jakobsson (Cham: Springer International Publishing), 510-519. doi: 10.1007/978-3-319-70278-0_32

Ananny, M., and Crawford, K. (2018). Seeing without knowing: limitations of the transparency ideal and its application to algorithmic accountability. New Media Soc. 20:973-989. doi: 10.1177/1461444816676645

Andreas, P. (2008). Sex, Drugs, and Body Counts: The Politics of Numbers in Global Crime and Conflict. Ithaca, NY: Cornell University Press

Antoniadis, P., and Martignoni, J. (2018). What Could Blockchain do for Community Networks. Retrieved from https://www.researchgate.net/profile/ Jens_Martignoni/publication/329990701_What_Could_Blockchain_do_ for_Community_Networks/links/5c27cc48458515a4c700acbc/What-CouldBlockchain-do-for-Community-Networks.pdf (accessed September 10, 2019).

Barsh, R. L. (1993). Measuring human rights: problems of methodology and purpose. Hum. Rights Quart. 15, 87-121. doi: 10.2307/762653

Bartoletti, M., Cimoli, T., Pompianu, L., and Serusi, S. (2018). "Blockchain for social good: a quantitative analysis," in Proceedings of the 4th EAI International Conference on Smart Objects and Technologies for Social Good. Goodtechs '18. (New York, NY: ACM), 37-42. doi: 10.1145/3284869.3284881

Bates, J. (2018). "Data cultures, power and the city," in Data and the City, eds R. Kitchin, T. P. Lauriault, and G. McArdle (New York, NY: Routledge). p. 189-200.

Bauwens, M., Kostakis, V., and Pazaitis, A. (2019). Peer to Peer: The Commons Manifesto. London: University of Westminster Press. doi: 10.16997/book33

Bauwens, M., and Pazaitis, A. (2019). P2P Accounting for Planetary Survival. P2P Foundation, Guerrilla Foundation and Schoepflin Foundation. Available online at: http://commonstransition.org/p2p-accounting-for-planetary-survival/

Bhushan, B., Khamparia, A., Sagayam, K. M., Sharma, S. K., Ahad, M. A., and Debnath, N. C. (2020). Blockchain for smart cities: a review of architectures, integration trends and future research directions. Sustain. Cities Soc. 61:102360. doi: $10.1016 /$ j.scs.2020.102360

Blockchain for Good: The Vision and Mission of the Dutch Blockchain Coalition (2018). Blockchain for Good: The Vision and Mission of the Dutch Blockchain Coalition. Dutch Blockchain Coalition.

Bollier, D. (2015). The Blockchain: a Promising New Infrastructure for Online Commons. Retrieved from http://www.bollier.org/blog/blockchain-promisingnew-infrastructure-online-commons (accessed September 10, 2019)

Bowker, G. C., and Star, S. L. (2000). Sorting Things Out: Classification and Its Consequences. Cambridge, MA: MIT Press. Available online at: https://mitpress. mit.edu/books/sorting-things-out

Bratton, B. H. (2016). The Stack: on Software and Sovereignty. Cambridge, MA: The MIT Press. doi: 10.7551/mitpress/9780262029575.001.0001

Campbell, D. T. (1979). Assessing the impact of planned social change. Eval. Program Plann. 2, 67-90. doi: 10.1016/0149-7189(79)90048-X

Cardullo, P., Di Feliciantonio, C., and Kitchin, R. (2019). The Right to the Smart City. Bingley Emerald Publishing Limited. doi: 10.1108/9781787691391

Cila, N., Gloerich, I., Ferri, G., de Waal, M., and Karpinski, T. (2020). "The blockchain and the commons: dilemmas in the design of local platforms," in Proceedings of the 2020. CHI Conference on Human Factors in Computing Systems - CHI '20 (Honolulu, HI: Association for Computing Machinery). doi: $10.1145 / 3313831.3376660$ this workshop, and Adam van Heerden, Irene Kamp, Merel Willemsen, Aspasia Beneti, Nina Fistal, Ben Schouten, Bodo Balasz, Geert Lovink, Chandar van der Zande, Shaun Lawson, Belen Barros Pena, Arthi Manohar, and Nicolai Brodersen Hansen for their participation. An account of the workshop was written up in Elsden et al. [2019b].

Cowley, R., Joss, S., and Dayot Y. (2017). The smart city and its publics: insights from across six UK Cities. Urban Res. Prac. 11, 53-77. doi: 10.1080/17535069.2017.1293150

Crawford, K., Lingel, J., and Karppi, T. (2015). Our metrics, ourselves: a hundred years of self-tracking from the weight scale to the wrist wearable device. Eur. J. Cult. Stud. 18, 479-496. doi: 10.1177/1367549415584857

Davidson, S., De Filippi, P., Potts J. (2016). Economics of blockchain. Econ. Environ. Innov. doi: 10.2139/ssrn.2744751

Davis, K., Fisher, A., Kingsbury, B., and Engle Merry, S. (2012b). Governance by Indicators: Global Power through Quantification and Rankings. (Law and Global Governance). Oxford; New York, NY: Oxford University Press. doi: 10.1093/acprof:oso/9780199658244.001.0001

Davis, K. E., Kingsbury, B., and Engle Merry, S. (2012a). Indicators as a technology of global governance. Law Soc. Rev. 46, 71-104. doi: 10.1111/j.1540-5893.2012.00473.x

De Filippi, P., and Wright, A. (2018). Blockchain and the Law: The Rule of Code. Cambridge, MA: Harvard University Press.

De Filippi, P., and Hassan, S. (2016). "Blockchain technology as a regulatory technology: from code is law to law is code. First Monday 21. doi: $10.5210 / \mathrm{fm} . v 21 \mathrm{i} 12.7113$

De Waal, M., and Dignum, M. (2017). The citizen in the smart city: how the smart city could transform citizenship. Inf. Technol. 59, 263-273. doi: 10.1515/itit-2017-0012

DuPont, Q. (2017). "Experiments in algorithmic governance: a history and ethnography of "The DAO," a failed decentralized autonomous organization," in Bitcoin and Beyond: Cryptocurrencies, Blockchains, and Global Governance. eds C. M. Verduyn (London: Routledge). doi: 10.4324/9781315211909-8

Elsden, C., Gloerich, I., Spaa, A., Vines, J., and de Waal, M. (2019b). Making the blockchain civic. Interactions 26, 60-65. doi: 10.1145/3305364

Elsden, C., Manohar, A., Briggs, J., Harding, M., Speed, C., and Vines, J. (2018). "Making sense of blockchain applications: a typology for HCI." in Proceedings of the 2018. CHI Conference on Human Factors in Computing Systems - CHI '18, 1-14. (Montreal, QC: ACM Press). doi: 10.1145/3173574.3174032

Elsden, C., Trotter, L., Harding, M., Davies, N., Speed, C., and Vines, J. (2019a). "Programmable donations: exploring escrow-based conditional giving," in Proceedings of the 2019 CHI Conference on Human Factors in Computing Systems (CHI '19). (New York, NY: ACM), 379. doi: 10.1145/3290605.3300609

Engle Merry, S. (2016). The Seductions of Quantification: Measuring Human Rights, Gender Violence, and Sex Trafficking. Chicago, IL: The University of Chicago Press. doi: 10.7208/chicago/9780226261317.001.0001

Espeland N. W., and Stevens, M. L. (2008). A sociology of quantification. Eur. J. Sociol. Arch. Eur. Sociol. 49, 401-436. doi: 10.1017/S0003975609000150

Farias, I., and Bender, T. (2010). Urban Assemblages: How Actor-Network Theory Changes Urban Studies. New York, NY: Routledge

Foth, M. (2017). “The promise of blockchain technology for interaction design," in Proceedings of the 29th Australian Conference on Computer-Human Interaction (OZCHI '17), eds A. Soro, D. Vyas, B. Ploderer, A. Morrison, J. Waycott, and M. Brereton (New York, NY: ACM), 513-517. doi: 10.1145/3152771.3156168

Foth, M., and Brynskov, M., and Ojala, T. (2015). Citizen's Right to the Digital City: Urban Interfaces, Activism, and Placemaking, eds M. Foth, M. Brynskov, and T. Ojala Singapore: Springer Singapore. doi: 10.1007/978-981-287-919-6

Frenken, K., van Waes, A., Smink, M., and van Est, R. (2017). A Fair Share Safeguarding Public Interests in the Sharing and Gig Economy. Utrecht: Rathenau Instituut 136

Friedman, B., Kahn, P. H., and Borning, A. (2008). "Value sensitive design and information systems," in The Handbook of Information and Computer Ethics (Hoboken, NJ: John Wiley \& Sons). 
Galen, D., Brand N., Boucherle, L., Davis, R. Do, N., El-Baz, B. et al. (2018). Blockchain for Social Impact: Moving Beyond the Hype. Stanford, CA: Center for Social Innovation, RippleWorks.

Gillespie, T. (2014). “The relevance of algorithms," in Media Technologies, eds T. Gillespie, P. J. Boczkowski, and K. A. Foot (Cambridge: The MIT Press), 167-194. doi: 10.7551/mitpress/9780262525374.003.0009

Gillespie, T. (2017). "Governance of and by platforms," in A. Handbook of Social Media, eds J. Burgess, T. Poell, and A. /Marwick (London: SAGE).

Hacking, I. (1999). The Social Construction of What? Cambridge, Mass: Harvard University Press.

Hawlitschek, F., Notheisen, B., and Teubner, T. (2018). The limits of trust-free systems: a literature review on blockchain technology and trust in the sharing economy. Electr. Commerce Res. Appl. 29, 50-63. doi: 10.1016/j.elerap.2018.03.005

Herian, R. (2018). "Blockchain and the distributed reproduction of capitalist class power," in MoneyLab Reader 2: Overcoming the Hype, eds I. Gloerich, G. Lovink, and P. de Vries (Amsterdam, NL: Institute of Network Cultures). p. 43-51. doi: 10.4324/9780429489815-2

Hwang, J. (2009). "U-City," in Handbook of Research on Urban Informatics: The Practice and Promise of the Real-Time City, eds M. Foth (Hershey, PA: IGI Global) 367-378

Kane, E. (2017). Is blockchain a general purpose technology? SSRN Electr. J. 1-27. doi: 10.2139/ssrn.2932585

Kewell, B., Adams, R., and Parry, G. (2017). Blockchain for Good? Strategic Change 26, 429-437. doi: 10.1002/jsc.2143

Kitchin, R. (2014). Making sense of smart cities: addressing present shortcomings. Camb. J. Regions Econ. Soc. 8, 131-136. doi: 10.1093/cjres/rsu027

Kitchin, R., Cardullo, P., and Di Feliciantonio, C. (2018). Citizenship, Justice and the Right to the Smart City. The Programmable City Working Paper 41. Available online at: https://osf.io/preprints/socarxiv/b8aq5. doi: 10.31235/osf.io/b8aq5

Kostakis, V., and Bauwens, M. (2014). Network Society and Future Scenarios for a Collaborative Economy. New York, NY: Springer.

Lefebvre, H., Kofman, E., and Lebas, E. (1996). Writings on Cities. Oxford: Blackwell Publishers.

Lessig, L. (1997). The constitution of code: limitations on choice-based critiques of cyberspace regulation. CommLaw Conspectus 5:12.

Lipsey, R. G., Carlaw, K. I., and Bekar, C. T. (2005). Economic Transformations: General Purpose Technologies and Long-Term Economic Growth. OUP Oxford.

Maréchal, N. (2018). "The data paradox: how the war on poverty became a war on the poor," in MoneyLab Reader \#2: Overcoming the Hype, eds I. Gloerich, G. Lovink, and P. de Vries. (Amsterdam, NL: Institute of Network Cultures).

Muller, J. Z. (2018). The Tyranny of Metrics. Princeton: Princeton University Press

Nakamoto, S. (2008). Bitcoin: A Peer-to-Peer Electronic Cash System. Available online at: https://bitcoin.org/bitcoin.pdf

Nissen, B., Pschetz, L., Murray-Rust, D., Mehrpouya, H. Oosthuizen, S., and Speed, C. (2018). "GeoCoin: supporting ideation and collaborative design with smart contracts," in Proceedings of the 2018 CHI Conference on Human Factors in Computing Systems (CHI '18). (New York, NY: ACM), 163. doi: $10.1145 / 3173574.3173737$

Nissen, B., Symons, K., Tallyn, E., Speed, C., Maxwell, D., and Vines, J. (2017). "New value transactions: understanding and designing for distributed autonomous organisations," in Proceedings of the 2017 ACM Conference Companion Publication on Designing Interactive Systems (DIS '17 Companion). (New York, NY: ACM), 352-355. doi: 10.1145/3064857.30 64862

O’Dwyer, R. (2019). “The Bank of Facebook.” Institute of Network Cultures Blog (blog). Available online at: https://networkcultures.org/blog/2019/06/19/ rachel-o-dwyer-the-bank-of-facebook/ (accessed June 19, 2019).

Ølnes, S., Ubacht, J., and Janssen, M. (2017). Blockchain in government: benefits and implications of distributed ledger technology for information sharing. Govern. Inform. Quart. 34, 355-364. doi: 10.1016/j.giq.2017. 09.007

Pazaitis, A., De Filippi, P., and Kostakis, V. (2017). Blockchain and value systems in the sharing economy: the illustrative case of backfeed. Technol. Forecast. Soc. Change 125, 105-115. doi: 10.1016/j.techfore.2017.05.025

Pisa, M., and Juden, M. (2017). Blockchain and Economic Development: Hype vs. Reality. Center for Global Development. Available online at: https://www. cgdev.org/publication/ blockchain-and-economic-development-hype-vsreality
Pitt, J., and Diaconescu, A. (2014). “The algorithmic governance of common-pool resources," in From Bitcoin to Burning Man and Beyond: The Quest for Identity and Autonomy in a Digital Society, eds J. H. Clippinger and D. Bollier (Amherst, MA: ID3 and Off the Common Books).

Porter, T. M. (1995). Trust in Numbers: The Pursuit of Objectivity in Science and Public Life. Princeton, NJ: Princeton University Press. doi: $10.1515 / 9781400821617$

Reijers, W., Wuisman, I., Mannan, M., De Filippi, P., Wray, C., Rae-Looi, V., et al. (2018). Now the code runs itself: on-chain and off-chain governance of blockchain technologies. Topoi. doi: 10.1007/s11245-018-9626-5. [Epub ahead of print].

Reshef Kera, D., Šourek, P., Kraiński, M., Reshef, Y., Corchado Rodríguez, J. M., and Knobloch, I. M. (2019). "Lithopia: prototyping blockchain futures," in Extended Abstracts of the (2019). CHI Conference on Human Factors in Computing Systems (CHI EA '19), (New York, NY: ACM), 6.

Rozas, D., Tenorio-Fornés, A., Díaz-Molina, S., and Hassan, S. (2018). When ostrom meets blockchain: exploring the potentials of blockchain for commons governance. SSRN Electr. J. 3272329. doi: 10.2139/ssrn.3272329

Sapienza, P., and Zingales, L. (2012). A trust crisis: a trust crisis. Int. Rev. Finance 12, 123-131. doi: 10.1111/j.1468-2443.2012.01152.x

Scott, J. C. (1998). Seeing Like a State: How Certain Schemes to Improve the Human Condition Have Failed. New Haven, CT: Yale University Press.

Sennett, R. (1970). The Uses of Disorder : Personal Identity and City Life. New York, NY: Norton.

Sennett, R. (2006). The Open City, Urban Age. Newspaper Essay, November, Berlin.

Sennett, R. (2018). Building and Dwelling: Ethics for the City. Farrar, Straus and Giroux.

Shen, C., and Pena-Mora, F. (2018). Blockchain for cities - a systematic literature review. IEEE Access 6, 76787-76819. doi: 10.1109/ACCESS.2018.2880744

Srnicek, N. (2017). Platform Capitalism. New York, NY: John Wiley \& Sons

Sultan, K., Ruhi, U., and Lakhani, R. (2018). Conceptualizing Blockchains: Characteristics \& Applications. Lisbon: Curran Assocites, Inc, 49-57.

Sun, J., Yan, J., and Zhang, K. Z. K. (2016). Blockchain-based sharing services: What blockchain technology can contribute to smart cities. Finan. Innov. 2:26. doi: 10.1186/s40854-016-0040-y

Szabo, N. (2014). "Unenumerated: The Dawn of Trustworthy Computing." Unenumerated (blog). Available online at: http://unenumerated.blogspot.com/ 2014/12/the-dawn-of-trustworthy-computing.html (accessed December 11, 2014)

Terranova, T., and Fumagalli, A. (2015). "Financial capital and the money of the common: the case of commoncoin," in MoneyLab Reader: An Intervention in Digital Economy, eds G. N., Tkacz, and P. de Vries. (Amsterdam: Institute of Network Cultures).

van der Graaf, S., (2018). In waze we trust: algorithmic governance of the public sphere. Media Commun. 6:153. doi: 10.17645/mac.v6i4.1710

Van Dijck, J., Poell, T., and de Waal, M. (2018). The Platform Society. Public Values in a Connective World. Oxford: Oxford University Press

van State, R. (2018). Ongevraagd advies Over de Effecten van de Digitalisering Voor de Rechtsstatelijke Verhoudingen. Available online at: https://www.raadvanstate. nl/@112661/w04-18-0230/

Wernimont, J. (2018). Numbered Lives: Life and Death in Quantum Media. Cambridge, MA: MIT Press. doi: 10.7551/mitpress/11455.001.0001

Wright, A., and De Filippi, P. (2018). Decentralized Blockchain Technology and the Rise of Lex Cryptographia. SSRN Scholarly Paper ID 2580664. Rochester, NY: Social Science Research Network. Available online at: https://papers.ssrn.com/ abstract=2580664. doi: 10.2139/ssrn.2580664

Yeung, K. (2018). Algorithmic regulation: a critical interrogation. Regul. Govern. 12, 505-523. doi: 10.1111/rego. 12158

Conflict of Interest: The authors declare that the research was conducted in the absence of any commercial or financial relationships that could be construed as a potential conflict of interest.

Copyright (c) 2020 Gloerich, De Waal, Ferri, Cila and Karpinski. This is an openaccess article distributed under the terms of the Creative Commons Attribution License (CC BY). The use, distribution or reproduction in other forums is permitted, provided the original author(s) and the copyright owner(s) are credited and that the original publication in this journal is cited, in accordance with accepted academic practice. No use, distribution or reproduction is permitted which does not comply with these terms. 